\title{
Шыхалиев Р.Г.
}

Институт Информационных Технологий НАНА, Баку, Азербайджан ramiz@science.az

\section{ИССЛЕДОВАНИЕ СОВРЕМЕННОГО СОСТОЯНИЯ ПРИМЕНЕНИЯ МЕТОДОВ МАШИННОГО ОБУЧЕНИЯ В НЕФТЕГАЗОВОЙ ОТРАСЛИ}

Поступила: 10.03.2020

Исправлена: 17.03.2020

Принята: 30.03 .2020

\begin{abstract}
Добыча, переработка и доставка продуктов нефти и газа требуют больших затрат. Поэтому основными задачами нефтегазовой отрасли, которые необходимо решать, являются повышение производительности добычи нефти и газа и минимизация затрат на добычу, переработку и доставку продуктов к конечным потребителям. При решении этих задач появляется множество проблем, таких как проблемы разведки и добычи нефти и газа, обнаружение аномалий при эксплуатациии буровых установок, инфраструктурных рисков в нефтепроводах, прогнозирование характеристик скважсин, минимизачия затрат на добычу и транспортировку нефти и газа, обнаружение утечек при транспортировке нефти и газа по проводам, оценка и управление рисками, прогнозирование волатильности цен на нефть и т.д. Решение большинства проблем традиционными методами анализа данных не представляется возможным, так как процессы нефтегазовой отрасли являются недетерминированными из-за их нелинейного характера, а также генерируют очень большие объемы данных. Поэтому в последнее десятилетие для решения проблем нефтегазовой отрасли применяются методы, основанные на искусственном интеллекте, в частности на методах машинного обучения (МO). В этой статье представлен обзор литературы по применению методов МО для решения различных задач нефтегазовой отрасли, что позволит определить потенцииал методов МО и более широко внедрять их в нефтегазовую отрасль.
\end{abstract}

Ключевые слова: нефтегазовая отрасль, искусственный интеллект, методы машинного обучения, метод опорных векторов, искусственные нейронные сети, глубокое обучение, генетические алгоритмы.

\section{Введение}

Несмотря на разработку альтернативных источников энергии, сегодня в мире нефть и газ являются основными энергоносителями. Поэтому нефть все еще является одним из наиболее потребляемых товаров в мире и влияет на нашу повседневную жизнь. Вместе с тем постоянно растет спрос на нефть и газ, и основными задачами, стоящими перед нефтегазовой отраслью, являются повышение производительности добычи нефти и газа и минимизация затрат на переработку и доставку продуктов к конечным потребителям [1].

Известно, что в нефтегазовой отрасли всегда имелось и сегодня имеется множество проблем. Эти проблемы в основном связаны с повышением эффективности бурения скважин и добычи нефти и газа, безопасностью, обслуживанием в нефтегазовой отрасли и т.д. Например, при бурении скважин в сложных условиях требуются большие капитальные вложения на строительство скважин, и для оптимизации бурения точное прогнозирование параметров бурения, например скорости бурения, является очень важным.

В нефтегазовой отрасли имеется множество процессов, которые генерируют огромное количество данных. В результате этого появляется множество проблем, связанных с их обработкой, таких как согласование данных различных источников из-за различия форматов, обеспечение безопасности потоков данных при сборе и передаче, анализ больших данных и т.д. Решение этих и других проблем может привести к повышению эффективности добычи и улучшению планирования нефтегазовой отрасли и т.п. 
Для решения указанных выше проблем сегодня используются новые подходы, основанные на методах искусственного интеллекта (ИИ) - методы машинного обучения. Основными целями МО являются прогнозирование, кластеризация, извлечение ассоциативных правил и поддержка принятия решений на основе полученной информации [2]. Использование методов МО позволит решить проблемы нефтегазовой отрасли, связанные с повышением производительности добычи, а также минимизацией затрат на добычу и транспортировку нефти и газа. Методы МО также могут быть применены для прогнозирования различных параметров бурения скважин и т.д.

В последнее время методы МО стали применяться при анализе, прогнозе, управлении и оптимизации процессов нефтегазовой отрасли. В литературе имеется ряд работ, посвященных использованию методов МО в нефтегазовой отрасли. МО позволяет разрабатывать более точные методы диагностики, мониторинга, прогнозирования и оптимизации бурения в реальном времени при минимальных затратах. Вместе с тем методы МО используются для решения отдельных конкретных задач нефтегазовой отрасли, таких как производство и управление. Несмотря на это, есть необходимость в исследовании методов МО, чтобы раскрыть их потенциал для широкого применения в нефтегазовой отрасли. Методами МО, которые могут быть применены для решения задач нефтегазовой отрасли, являются метод опорных векторов (МOB) [3], искусственные нейронные сети (ИНС) [4], глубокое обучение (ГО) [5], генетические алгоритмы (ГА) [6] и т.д.

Целью данной статьи являются обзор работ по применению методов МО для решения задач нефтегазовой отрасли и анализ проблем их применения. Для эффективного использования методов МО в нефтегазовой отрасли требуется глубокое понимание как методов МО, так и процессов нефтегазовой отрасли.

\section{Проблемы нефтегазовой отрасли}

Современная нефтегазовая отрасль является очень большой, технологически сложной и капиталоемкой отраслью и включает в себя три ключевых сектора. Первый сектор включает разведку и добычу нефти и газа, то есть состоит из поиска подземных и подводных месторождений нефти и газа, а также бурение разведочных скважин и скважин для добычи нефти и газа. Второй сектор состоит из транспортировки, хранения и переработки нефти и газа, то есть после добычи нефти и газа их необходимо транспортировать на нефтеперерабатывающие заводы и в газовые хранилища, которые обычно находятся удаленно от места добычи. Для транспортировки нефти и газа могут быть использованы различные средства, такие как танкеры, трубопроводы, железнодорожные и автоцистерны. Третий сектор включает в себя маркетинг и коммерческое распространение продуктов переработки сырья среди потребителей и конечных пользователей. Продуктами переработки сырья могут быть природный газ, дизельное топливо, бензин, смазочные материалы, керосин, авиационное топливо, асфальт, мазут, сжиженный газ и т.д.

Каждый из вышеуказанных секторов нефтегазовой отрасли имеет специфические проблемы, которые требуют принятия соответствующих решений [7]. К основным проблемам нефтегазовой отрасли могут быть отнесены проблемы, связанные с увеличением скорости бурения скважин, повышением эффективности добычи, обнаружением аномалий при эксплуатации буровых установок, безопасностью и обслуживанием, прогнозом цен и т.д. Для решения этих проблем могут быть использованы различные технологии, такие как оцифровка месторождений, оптимизация буровых работ в режиме реального времени, использование нанотехнологий, беспроводных сенсорных сетей для облегчения измерений, моделирование пласта и диагностики, а также интеллектуальных технологий, в частности для обработки и анализа данных. 


\section{Применение машинного обучения в нефтегазовой отрасли}

Методы МО могут быть классифицированы следующим образом: контролируемое обучение (supervised learning) - классификация; неконтролируемое обучение (unsupervised learning) - кластеризация; частично контролируемое обучение (semi-supervised learning) и обучение с подкреплением (reinforcement learning) [8].

В контролируемом алгоритме обучения используется обучающий набор данных (примеров), в которых данные имеют известные атрибуты и связанные с ними ответы, представляющие класс данных. Используя обучающий набор, алгоритм изучает шаблоны в данных и обобщает их, чтобы правильно классифицировать новые входные данные. В неконтролируемом алгоритме обучения не имеется ответов, связанных с атрибутами данных, a алгоритм идентифицирует сходство между входными данными и классифицирует их на основе этих сходств. Алгоритм обучения с частичным контролированием обучается с помощью обучающего набора данных, который содержит как помеченные (данные, относящиеся к конкретным классам), так и непомеченные данные (данные, не относящиеся к конкретным классам). При обучении с подкреплением алгоритм по обратной связи получает информацию об ошибках, но при этом не получает инструкцию по исправлению этих ошибок, и поэтому алгоритм, используя метод проб и ошибок, постоянно обучается.

Одним из первых применений МО в нефтегазовой отрасли было прогнозирование параметров бурения скважин [9]. В этой работе автор использовал ИНС для прогнозирования параметра износа буровой коронки, а в работе [10] ИНС была использована для прогнозирования скорости бурения скважин (таблица 1).

Методы МО также могут применяться для прогнозирования других параметров бурения, таких как давление закачивания бурового раствора [11], плотность бурового раствора [12], а также для оптимизации параметров бурения [13] и т.д. Кроме того, МО был эффективно использован для моделирования и профилактического обслуживания резервуаров. В частности, методы МО были использованы для определения оптимального времени технического обслуживания резервуаров, чтобы избежать сбоев.

В работе [14] проведен SWOT (Strengths, Weaknesses, Opportunities, Threats)-анализ по использованию MO в нефтегазовой отрасли. В результате анализа автор делает вывод о том, что для применения МО в решении критически важных задач в нефтегазовой отрасли должны быть использованы новейшие технологические разработки и созданы совместные команды из ученых по анализу данных и экспертов нефтегазовой отрасли.

Наличие в газопроводе твердых веществ при больших концентрациях и давлениях может разрушить механическое оборудование инфраструктуры газопроводов. Поэтому появляется необходимость в проведении в реальном времени мониторинга наличия твердых веществ в газопроводе. Для решения этой задачи в работе [15] предлагается методология мониторинга в реальном времени газового потока в газопроводе. Мониторинг заключается в оперативном измерении скорости и концентрации потока твердых веществ, а также перепада давления и скорости газа в многофазном газовом потоке с использованием технологии акустического зондирования в сочетании с методами обработки сигналов и алгоритмов МО. Для установления связи между измеряемыми величинами и акустическим сигналом используются обычные ИНС. Однако обычные ИНС не могут фиксировать динамику генерируемого во времени акустического сигнала. Поэтому авторами была предложена модификация обычных ИНС на так называемую нейронную сеть с временной задержкой (Time-Delay Neural Network - TDNN), которая и использовалась для фиксировании такой динамики. Была сравнена производительность классической модели ИНС и модели TDNN, и полученные результаты продемонстрировали значительную перспективу развития системы количественного мониторинга газового потока в реальном времени. 
Применение методов МО в нефтегазовой отрасли

\begin{tabular}{|c|c|c|}
\hline Автор(ы), год & Метод, алгоритм & Цель \\
\hline Arehart, R., 1990 & ИНС & $\begin{array}{l}\text { прогнозирование параметра износа } \\
\text { буровой коронки }\end{array}$ \\
\hline Bilgesu H. et.al., 1997 & ИНС & $\begin{array}{l}\text { прогнозирование скорости бурения } \\
\text { скважин }\end{array}$ \\
\hline Yulmaz S., et.al., 2002 & ИНС & оптимизация параметров бурения \\
\hline Velez-Langs O., 2005 & $\Gamma A$ & $\begin{array}{l}\text { разработка нефтяных месторождений; } \\
\text { планирование добычи; определение } \\
\text { характеристик коллекторов и } \\
\text { сейсмической инверсии }\end{array}$ \\
\hline Jin H., et.al., 2014 & LS-SVM & обнаружение уровней утечек в газопроводе \\
\hline Wang Y., et.al., 2015 & ИНС & $\begin{array}{l}\text { прогнозирование давления закачивания } \\
\text { бурового раствора }\end{array}$ \\
\hline Ahmadi M.A., 2016 & LS-SVM & $\begin{array}{l}\text { прогнозирование плотности бурового } \\
\text { раствора }\end{array}$ \\
\hline $\begin{array}{l}\text { Kristjanpoller W., et.al., } \\
2016\end{array}$ & ANN-GARCH & $\begin{array}{l}\text { прогнозирование волатильности цен на } \\
\text { нефть }\end{array}$ \\
\hline Qiao Y., et.al., 2017 & PSO, LS-SVM & прогнозирование добычи нефти и газа \\
\hline Panja P., et.al., 2018 & $\begin{array}{l}\text { SVM, ИНС и } \\
\text { RSM } \\
\end{array}$ & $\begin{array}{l}\text { прогнозирование добычи углеводородов из } \\
\text { гидравлически разрываемых скважин }\end{array}$ \\
\hline Li H., et.al., 2018 & VAE, CNN, LSTM & определение характеристик недр \\
\hline $\begin{array}{l}\text { Aminu K.T., et.al., } \\
2019\end{array}$ & ИНС & $\begin{array}{l}\text { мониторинг в реальном времени наличия } \\
\text { твердых веществ в газопроводе }\end{array}$ \\
\hline $\begin{array}{l}\text { Paltrinieria N., et.al., } \\
2019\end{array}$ & $\Gamma \mathrm{HC}$ & оценка рисков в нефтегазовой отрасли \\
\hline $\begin{array}{l}\text { Rahmanifard, H., et.al., } \\
2019\end{array}$ & $\begin{array}{c}\text { ГА, ОРЧ и } \\
\text { дифференциальна } \\
\text { я эволюция }\end{array}$ & $\begin{array}{l}\text { оптимизация скорости добычи нефти; } \\
\text { определение минимального давления } \\
\text { смешиваемости и объема секвестрации } \\
\mathrm{CO}_{2}\end{array}$ \\
\hline $\begin{array}{l}\text { Abdullayeva F.D., } \\
\text { Imamverdiyev Y.N., } \\
2019\end{array}$ & CNN-LSTM & $\begin{array}{l}\text { прогнозирование временных рядов добычи } \\
\text { нефти }\end{array}$ \\
\hline $\begin{array}{l}\text { Imamverdiyev Y., } \\
\text { Sukhostat L., } 2019\end{array}$ & $1 \mathrm{D}-\mathrm{CNN}$ & $\begin{array}{l}\text { классификация геологических фаций } \\
\text { скважин }\end{array}$ \\
\hline
\end{tabular}

Прогнозирование факторов, влияющих на добычу, существенно для достижения эффективности добычи нефти и газа. В работе [16] была предложена модель прогнозирования добычи нефти и газа, которая включает метод оптимизации роя частиц (ОРЧ - PSO (Particle Swarm Optimization)) и метод опорных векторов наименьших квадратов (LS-SVM - Least-Squares Support-Vector Machines). Поскольку алгоритм ОРч легко попадает в локальный оптимум, то авторы используют критерий преждевременной сходимости, основанный на информации о разнообразии популяций. Это позволяет выбрать начальную популяцию и повысить общее качество популяции и способность к глобальной оптимизации. Для прогнозирования добычи нефти и газа и анализа соответствующих факторов, влияющих на добычу, используется PSO LS-SVM-модель. Используя реальные данные нефтегазовых компаний и моделирование, проведенное в MATLAB, авторы смогли проанализировать факторы, влияющие на добычу, и построить 
модель точного прогнозирования, которая была обучена с высокой скоростью.

Для прогнозирования добычи углеводородов из гидравлически разрываемых скважин в работе [17] используются модели SVM, ИНС и RSM (Response Surface Model). Авторы сравнивают модели LS-SVM и ИНC с моделью RSM, используя полиномиальные уравнения второго порядка, чтобы прогнозировать добычу из сланцев. В качестве входных факторов в модели используются восемь параметров, такие как проницаемость пласта, начальное отношение растворенного газа и нефти, сжимаемость породы, относительная проницаемость газа, наклон газового фактора, начальное пластовое давление, пластовое давление в забое скважины и расстояние между гидроразрывами. Согласно результатам тестов модель LS-SVM продемонстрировала наивысшую точность в прогнозировании соотношения газ-нефть.

Использование методов ГО позволяет обнаруживать закономерности в наборах данных и извлекать полезную информацию. Их применение в нефтегазовой отрасли позволит обнаружить аномалии при эксплуатации буровых установок, просчитать инфраструктурные риски в нефтепроводах, прогнозировать характеристики скважин и т.д. Например, для определения характеристик недр в работе [18] были предложены две модели ГО прогнозирования ядерного магнитного резонанса по распределению времени Т2 по обычным каротажам скважин. Для этого авторы использовали вариационный автокодер (VAE - Variational Autoencoder) в сочетании со сверточной нейронной сетью (CNN Convolutional Neural Network), чтобы эффективно извлечь признаки из распределения Т2, а сеть с долговременной кратковременной памятью (LSTM - Long Short-Term Memory), чтобы извлечь краткосрочные и долгосрочные зависимости из распределения Т2. Для обучения и тестирования моделей были использованы данные, полученные из Баккенской сланцевой формации. Результаты показали, что точность прогноза моделей в целом была хорошей и обе модели были устойчивы к шуму. А в работе [19] для классификации геологических фаций в скважинах предлагается модель, основанная на ГО. Классификация фаций осуществляется путем изучения литологических свойств горных пород, характерных для современных отложений, накапливающихся при определенных физико-географических условиях. Для решения этой задачи авторы предлагают модель, называемую 1D-CNN, которая обучается различным алгоритмам оптимизации. В качестве входных данных модели рассматриваются фотоэлектрический эффект, гамма-излучение, каротаж удельного сопротивления, разность пористости нейтронной плотности, средняя пористость нейтронной плотности и геологические ограничивающие переменные. Приемлемая точность и использование традиционных данных каротажа скважин являются основными преимуществами предлагаемой модели. Предложенная модель была сравнена с такими моделями, как RNN (Recurrent Neural Network), LSTM, SVM, KNN, и показала более точные результаты по сравнению с ними.

Одной из проблем нефтегазовой отрасли является быстрое выявление снижения объема полезных флюидов и увеличения объема бесполезных флюидов, которое влияет на нефтеотдачу скважин. Для решения этой проблемы в работе [20] авторы предлагают метод прогнозирования добычи нефти с использованием глубоких нейронных сетей на основе дебитных данных скважин. В частности, предлагается гибридная модель прогнозирования временных рядов добычи нефти, основанная на комбинации сетей CNN и LSTM, называемая CNN-LSTM. Архитектура предлагаемой авторами модели является иерархической: сначала слой CNN-модели применяется к текущему временному окну, а затем связь между временными окнами прогнозируется путем применения LSTM. Для оценки эффективности модели используется набор данных QRI, a точность прогнозирования проверяется с использованием метрики RMSLE (Root Mean Squared Logarithmic Error). 
Оценка и управление рисками в критически важных промышленных отраслях, к которым относится нефтегазовая отрасль, играют ключевую роль. Требуются постоянная оценка и управление рисками, для которых подходящим является использование методов МO. В работе [21] был предложен метод оценки рисков в нефтегазовой отрасли на основе МO. В частности, была разработана и испытана модель глубокой нейронной сети (ГНС) для оценки рисков в буровых установках нефти и газа. Результаты тестов показали приемлемую точность прогноза ГНС и способность к преодолению трудностей при оценке рисков.

В области использования методов ГА в нефтегазовой отрасли, особенно в сфере разведки и добычи нефти и газа, имеются ряд работ. В [22] автор проводит обзор некоторых работ, посвященных разработке нефтяных месторождений, планированию добычи, определению характеристик коллекторов и сейсмической инверсии. Использование методов ГА позволяет повысить эффективность разведки и добычи нефти и газа.

ИИ широко применяется в системах поддержки принятия решений в различных отраслях промышленности, в том числе в нефтегазовой отрасли. Разработанные на основе ИИ системы способны постоянно учиться, тем самым непрерывно совершенствуясь. В работе [23] было рассмотрено применение методов ИИ в некоторых областях нефтегазовой отрасли, например, моделирование коллектора, распознавание сейсмических образов, определение характеристик коллектора, прогноз проницаемости и пористости пластов, диагностика бурового долота, оптимизация скважин и т.д.

В работе [24] было проанализировано несколько методов ИИ, применяемых в решении некоторых задач нефтегазовой отрасли, таких как оптимизация скорости добычи нефти, определение минимального давления смешиваемости и объема секвестрации $\mathrm{CO}_{2}$. При этом особое внимание уделялось эвристическим методам оптимизации, таким как ГА, ОРЧ и дифференциальная эволюция.

При транспортировке нефти и газа по проводам очень важно обнаружение утечек. В работе [25] был использован LS-SVM для обнаружения уровней утечек в газопроводе на основе метода акустических волн. Авторы проводили эксперименты на испытательном стенде, состоящем из газопровода, компрессоров, клапанов для регулирования, извлечения акустических данных, и системах моделирования утечки для моделирования различных уровней утечки. При этом акустические сигналы были собраны при нормальных, малых, средних и больших утечках. Результаты эксперимента показали, что модель LS-SVM может идентифицировать различные уровни утечки на основе собранных сигналов в течение короткого времени и с высокой точностью.

Для прогнозирования волатильности цен на нефть в работе [26] авторами был предложен метод ИНС-обобщенной авторегрессионной условной гетероскедастичности (ОФУГ) (ANN-GARCH - Artificial Neural Network- Generalized AutoRegressive Conditional Heteroskedasticity). В этом методе ИНС использует результаты прогноза ОФУГ и данные финансовых временных рядов для определения нестабильности цен на нефть. Авторы использовали разницу курсов и индексов фондового рынка для улучшения прогноза цен на сырую нефть. Для анализа и улучшения прогнозирующей способности модель была применена в трех разных областях прогнозирования. Кроме того, критерий оценки функции потерь был использован для определения хода прогнозирования волатильности. Наборы данных, проанализированные в этом исследовании, были получены из спотовых цен на нефть и фьючерских цен на нефть Bloomberg за период с июля 2002 года по май 2014-го. Результаты показали, что предлагаемый метод улучшил прогнозы модели ОФУГ в отношении изменчивости спотовой цены на нефть и фьючерсов с использованием 21дневного окна. 


\section{Заключение}

В данной статье проведен обзор работ по использованию методов МО для решения некоторых задач нефтегазовой отрасли. Методы МО позволяют извлечь информацию из большого объема данных, чтобы решить задачи прогнозирования, кластеризации, извлечения ассоциативных правил и поддержки принятия решений. Однако традиционные методы анализа данных являются менее точными, чем методы MO, а при больших объемах данных вообще не подходят для решения указанных задач.

Были рассмотрены такие методы МО, как МОВ, ИНС, ГО, ИНС, ГА и т.д., которые были применены к решению различных задач нефтегазовой отрасли. Обзор охватывает такие проблемы нефтегазовой отрасли, как проблемы разведки и добычи нефти и газа, обнаружение аномалий при эксплуатации буровых установок, инфраструктурных рисков в нефтепроводах, прогнозирование характеристик скважин, минимизация затрат на добычу и транспортировку нефти и газа, обнаружение утечек при транспортировке нефти и газа по проводам, оценка и управление рисками, прогнозирование волатильности цен на нефть и.т.д.

Анализ рассмотренных работ показал, что при решении различных проблем нефтегазовой отрасли методы МО в основном применялись для решения задач прогнозирования (табл.). При детальном анализе этих решений можно видеть, что для решения одних и тех же проблем используются различные методы МО, данные и масштабы. С точки зрения доказательства эффективности применяемых в нефтегазовой отрасли методов МО необходимо полностью исследовать и экспериментировать их в реальности.

\section{Благодарность}

Данная работа выполнена при финансовой поддержке Фонда науки Государственной нефтяной компании Азербайджана SOCAR - Контракт № 23 LR-AMEA.

\section{Литература}

1. Chima C.M., Hills D. Supply-chain management issues in the oil and gas industry // Journal of Business and Economics Research, 2007, vol. 5, no. 6, pp. 27-36.

2. Mohammed M., Khan M.B., Bashier E.B.M. Machine learning: algorithms and applications, CRC Press, 2017, 46 p.

3. Evgeniou T., Pontil M. Support Vector Machines: Theory and Applications / Machine Learning and Its Applications, Advanced Lectures, 2001, pp. 249-257.

4. Patterson D.W. Artificial neural networks: Theory and Applications // Prentice Hall, 1996, 477 p.

5. Pouyanfar S., Sadiq S., Yan Y., Tian H., Tao Y., Reyes M. P., Shyu M.L, Chen S., Chen S.C., Iyengar S.S. A Survey on Deep Learning: Algorithms, Techniques and Applications // ACM Computing Surveys, 2018, vol. 51, no.5, 36 p.

6. Bodenhofer U. Genetic Algorithms: Theory and Applications // Lecture Notes, 2003, 126 p.

7. Tsegha E. Assessing the challenges and opportunities in the oil and gas industry // Academic Journal of Interdisciplinary Studies, 2013, vol. 2, no. 12, pp. 129-136.

8. Balaji K., Rabiei M., Suicmez V., Hakan C. C., Agharzeyva Z., Tek S., Bulut U., Temizel C. Status of Data-Driven Methods and their Applications in Oil and Gas Industry / SPE Europec featured at 80th EAGE Conference and Exhibition, 2018, 20 p.

9. Arehart R. Drill-bit diagnosis with neural networks // SPE Computer Applications, 1990, vol. 2, no. 4, pp. 24-28.

10. Bilgesu H., Tetrick, L., Altmis, U., Mohaghegh, S., Ameri, S. A new approach for the prediction of rate of penetration (ROP) values / SPE Eastern Regional Meeting, 1997, pp. 175-180.

11. Wang Y., Salehi, S. Application of real-time field data to optimize drilling hydraulics using neural network approach // Journal of Energy Resources Technology, 2015, vol. 137, no. 6, 9 p. 
12. Ahmadi M.A. Toward reliable model for prediction Drilling Fluid Density at wellbore conditions: a LSSVM model // Neurocomputing, 2016, vol. 211, pp. 143-149.

13. Yılmaz S., Demircioglu, C., Akin, S. Application of artiaшcial neural networks to optimum bit selection // Computers \& Geosciences, 2002, vol. 28, no. 2, pp. 261-269.

14. Hajizadeh Y. Machine learning in oil and gas; a SWOT analysis approach // Journal of Petroleum Science and Engineering, 2019, vol. 176, pp. 661-663.

15. Aminu K.T., McGlinchey D. Cowell A. Acoustic signal processing with robust machine learning algorithm for improved monitoring of particulate solid materials in a gas flowline // Flow Measurement and Instrumentation, 2019, vol. 65, pp. 33-44.

16. Qiao Y., Peng J., Ge L., Wang H. Application of PSO LS-SVM forecasting model in oil and gas production forecast / IEEE 16th International Conference on Cognitive Informatics \& Cognitive Computing, 2017, pp. 470-474.

17. Panja P., Velasco R., Pathak M., Deo M. Application of artificial intelligence to forecast hydrocarbon production from shales // Petroleum 2018, vol.4, no.1, pp.75-89.

18. Li H., Misra S. Long short-term memory and variational autoencoder with convolutional neural networks for generating nmr $\mathrm{t} 2$ distributions / IEEE Geoscience and Remote Sensing Letters, 2018, vol. 16, no. 2, pp. 192-195.

19. Imamverdiyev Y., Sukhostat L. Lithological facies classification using deep convolutional neural network // Journal of Petroleum Science and Engineering, v.174, March 2019, pp. 216-228.

20. Abdullayeva F.D., Imamverdiyev Y.N., Development of oil production forecasting method based on Deep Learning // Statistics, Optimization and Information Computing, 2019, vol. 7, pp. 826-839.

21. Paltrinieria N., Comfort L., Reniers G. Learning about risk: Machine learning for risk assessment // Safety Science, 2019, vol. 118, pp. 475-486.

22. Velez-Langs O. Genetic algorithms in oil industry: An overview // Journal of Petroleum Science and Engineering, 2005, vol. 47, no.1-2, pp. 15-22.

23. Bello O., Teodoriu, C., Yaqoob, T., Oppelt, J., Holzmann, J., Obiwanne, A. Application of artificial intelligence techniques in drilling system design and operations: a state of the art review and future research pathways / SPE Nigeria Annual International Conference and Exhibition, 2016, vol. 5, no. 2, pp. 121-139.

24. Rahmanifard H., Plaksina, T. Application of artificial intelligence techniques in the petroleum industry: a review // Artificial Intelligence Review, 2019, vol, 52, pp. 2295-2318.

25. Jin H., Zhang L., Liang W., Ding Q. Integrated leakage detection and localization model for gas pipelines based on the acoustic wave method // Journal of Loss Prevention in the Process Industries, 2014, vol. 27, pp. 74-88.

26. Kristjanpoller W., Minutolo M.C. Forecasting volatility of oil price using an artificial neural network-GARCH model // Expert Systems with Applications, 2016, vol. 65, pp. 233-241.

\section{UOT 004.853}

\section{Şıxəliyev Ramiz H.}

AMEA İnformasiya Texnologiyaları İnstitutu, Bakı, Azərbaycan ramiz@science.az

\section{Neft-qaz sənayesində maşın təlimi metodlarının tətbiqinin müasir vəziyyətinin tədqiqi}

Neft və qazın çıxarılması, emalı və məhsullarının çatdırılması baha başa gəlir. Buna görə neft və qaz hasilatının məhsuldarlığının artırılması, məhsulun emal edilməsi və son istehlakçılara çatdırması xərclərinin minimuma endirilməsi neft-qaz sənayesinin həll edilməli olan əsas məsələləridir. Bu məsələləri həll edərkən neft və qaz kəşfiyyatı, hasilat1 problemləri, qazma qurğularının istismarında anomaliyaların aşkar edilməsi, neft boru kəmərlərində infrastruktur 
risklərinin aşkarlanması, quyuların xüsusiyyətlərinin proqnozlaşdırılması, neft və qazın hasilatı, daşınması xərclərinin minimuma endirilməsi, boru kəmərləri vasitəsilə neft və qazın daşınması zamanı sızmaların aşkarlanması, risklərin qiymətləndirilməsi, idarə edilməsi, neft qiymətlərinin dəyişkənliyinin proqnozlaşdırması və s. kimi bir çox problemlər ortaya çıxır. Oksər problemlərin verilənlərin analizinin ənənəvi üsulları ilə həlli mümkün deyil, çünki neft və qaz sənayesi prosesləri qeyri-xətti xarakterinə görə qeyri-müəyyəndir və bu proseslər çox böyük həcmdə məlumat yaradır. Buna görə də son on ildə neft-qaz sənayesindəki problemlərin həlli üçün ədəbiyyatda süni intellektə əsaslanan metodlar, xüsusən də maşın təliminə (MT) əsaslanan metodlar təklif edilmişdir. Bu məqalədə neft-qaz sənayesinin müxtəlif problemlərini həll etmək üçün MT metodlarının tətbiqi ilə bağlı ədəbiyyatların icmalı verilib və bu, MT metodlarının potensialını müəyyən etməyə və neft-qaz sənayesində daha geniş tətbiq etməyə imkan verər.

Açar sözlor: neft-qaz sənayesi, süni intellekt, maşın talimi metodlarl, dəstəklayici vektor metodu, süni neyron şabəkalari, darin öyranma, genetik alqoritmlar.

\section{Ramiz H. Shikhaliyev}

Institute of Information Technology of ANAS, Baku, Azerbaijan

ramiz@science.az

\section{Research of the current state of machine learning methods application in the oil and gas industry}

The extraction, refining and delivery of oil and gas products is expensive. Therefore, the main tasks of the oil and gas industry that need to be addressed are to increase the productivity of oil and gas production and minimize the cost of processing and delivery of the products to end consumers. When solving these tasks, many problems arise, such as the problems of oil and gas exploration and production, detection of anomalies in the operation of drilling rigs, detection of infrastructure risks in oil pipelines, prediction of well characteristics, minimization of expenses on oil and gas production and transportation, and detection of leaks during oil transportation and gas pipelines, risk assessment and management, forecasting oil price volatility, etc. The solution to most problems by traditional methods of data analysis is not possible, since the processes of the oil and gas industry are non-deterministic due to their non-linear nature, and also these processes generate very large amounts of data. Therefore, in the last decade, to solve the problems of the oil and gas industry, the methods based on artificial intelligence, in particular, on machine learning (ML) methods, have been proposed in the literature. This article provides a review of the literature on the application of ML methods to solve various problems of the oil and gas industry, which allow to determine the potential of ML methods and more widely implement them in the oil and gas industry.

Keywords: oil and gas industry, artificial intelligence, machine learning methods, support vector method, artificial neural networks, deep learning, genetic algorithms. 